\title{
Cognição Numérica em Crianças com Transtornos Específicos de Aprendizagem
}

\author{
Paulo Adilson da Silva \\ Universidade Estadual Paulista Júlio de Mesquita Filho, SP, Brasil \\ Fabiana Silva Ribeiro \\ Escola de Psicologia da Universidade do Minho, Braga, Portugal \\ Flávia Heloísa Santos ${ }^{1}$ \\ Programa de Pós-Graduação em Psicologia do Desenvolvimento e Aprendizagem \\ da Universidade Estadual Paulista Júlio de Mesquita Filho, Bauru, SP, Brasil \\ Departamento de Psicologia Básica da Universidade do Minho, Braga, Portugal
}

\section{Resumo}

O objetivo do presente estudo foi investigar o desenvolvimento de sistemas específicos da Cognição Numérica (Senso Numérico - SN, Compreensão Numérica - CN, Produção Numérica - PN e Cálculo - CA) em crianças brasileiras com Transtornos Específicos de Aprendizagem. Participaram do estudo 72 crianças (36 meninos), entre 9 e 10 anos, cursando os $4^{\circ}$ ou $5^{\circ}$ Anos do Ensino Fundamental em escolas públicas da região centro-oeste do Estado de São Paulo, Brasil; divididas em três grupos: controle (CT, $N=42$ ), com perfil de Dislexia do Desenvolvimento (DL, $N=11$ ) e com perfil de Discalculia do Desenvolvimento combinada com dislexia (DDc, $N=19$ ). Todas apresentavam nível intelectual médio para faixa etária. Os Transtornos Específicos de Aprendizagem foram aferidos pelo Teste de Desempenho Escolar (Stein, 1994), no qual as crianças com DDc obtiveram necessariamente classificação 'inferior' no Teste de Aritmética. Os três grupos foram similares em SN. As crianças DL e DDc apresentaram prejuízos leves em CN. Contudo, crianças com DDc apresentaram prejuízos moderados em PN e de leve a moderado em CA, o que indica um comprometimento mais generalizado em Cognição Numérica quando comparadas aos outros grupos. Em conclusão, crianças com DDc apresentaram maiores prejuízos e defasagem em relação às crianças DL e com desenvolvimento típico nos diferentes sistemas da Cognição Numérica.

Palavras-chave: Transtornos Específicos de Aprendizagem; Discalculia do Desenvolvimento; Dislexia do Desenvolvimento; Cognição Numérica; crianças.

Endereço para correspondência: Universidade Estadual Paulista “Júlio de Mesquita Filho", Programa de Pós-Graduação em Psicologia do Desenvolvimento e Aprendizagem, Campus Bauru, Av. Engenheiro Luiz Edmundo Carrijo Coube, 14-01, Bauru, SP, Brasil 19806-173. Fone: + 5514 31036077. E-mail: flaviahs@psi. uminho.pt

Agradecemos especialmente aos pais e crianças pela participação e cooperação das escolas das cidades do oestepaulista do Estado de São Paulo. Estudo financiado pelo Acordo Internacional entre a Fundação de Amparo à Pesquisa do Estado de São Paulo (FAPESP) e Institut National de La Santé et de la Recherche Médicale (INSERM), processo $n^{\circ}$ 04/11.067-0; bolsistas: Juliana Molina $n^{\circ}$ 05/00595-8, Bruna Paschoalini ${ }^{\circ} 05 / 00594-$ 1, Rosana Satiko Kikuchi no 08/54971-2; Michele Cândida Frigério nº 05/00593-5; Ana Luiza Ribeiro Pereira $\operatorname{Dias}^{\circ}$ 2005/00592-9; Fabiana Silva Ribeiro nº8/54970-2; Paulo Adilson da Silva nº 05/60375-1. 


\title{
Numerical Cognition in Children with Specific Learning Disabilities
}

\begin{abstract}
The aim of this study was to investigate the development of specific domains of Numerical Cognition (Number Sense - NS, Number Comprehension - NC, Number Production - NP, and Calculation - CA) in Brazilian children with Specific Learning Disorders. The study included 72 children (36 boys), from 9-to 10-years-old, enrolled in 4th and 5th years of elementary school of public schools at countryside of Sao Paulo State, Brazil. They were divided into three groups: control (CT, $N=42)$, Developmental Dyslexia (DL, $N=11$ ) and Developmental Dyscalculia combined with dyslexia (DDc, $N=19$ ). All participants had intellectual level within the normal range, however, children from the last two groups had Learning Disorders classified by School Achievement Test - TDE (Stein, 1994) and DDc children received necessarily classification as 'inferior' in Arithmetic Test of TDE. The children did not differ in NS. DL and DDc children showed slight deficits in NC. However, DDc children had moderate in $\mathrm{NP}$ and mild to moderate deficits in CA, which indicates a more generalized impairment in Numerical Cognition. Furthermore, DDc children showed discrepancy in Numerical Cognition performance when compared to the other groups. Thus, children with Learning Disorders showed different performances in Numerical Cognition, although both groups had preserved SN, DDc children showed higher deficits and discrepancy in relation DL and typically developing children.
\end{abstract}

Keywords: Specific Learning Disabilities, Developmental Dyscalculia, Developmental Dyslexia, Numerical Cognition, children.

\section{Cognición Numérica en Niños con Trastornos Específicos de Aprendizaje}

\section{Resumen}

El objetivo de este estudio fue investigar el desarrollo de los sistemas específicos de la Cognición Numérica (el Sentido Numérico - SN, la comprensión numérica - CN, la producción numérica - PN y el Cálculo - CA) en niños brasileños con Trastornos Específicos de Aprendizaje. El estudio incluyó a 72 niños (36 chicos), con edad entre 9 y 10 años, estudiantes de los años 4to y 5 to de primaria en escuelas públicas de un pueblo del Estado de São Paulo, Brasil; divididos en tres grupos: control (CT, $N=42$ ), perfil de la Dislexia de Desarrollo (DL, $N=11$ ) y el perfil de la Discalculia del Desarrollo combinado con dislexia (DDc, $N=19)$. Todos tenían el nivel intelectual dentro del rango medio. Los trastornos del aprendizajes e evaluaron a través de la Prueba de Rendimiento Académico (Stein, 1994), en el que los niños con DDc obtuvieron una clasificación necesariamente "inferior" en la prueba de aritmética. Los tres grupos fueron similares en SN. Los niños con DL y DDc mostraron ligeras pérdidas en CN. Sin embargo, los niños con DDc sufrieron daños moderados en PN y de leves a moderados en CA, por lo tanto, exhibieron un deterioro más generalizado en la cognición numérica en comparación a los otros grupos. En conclusión, los niños con DDc presentaron mayores perjuicios do que niños con DL o desarrollo típico de los diferentes sistemas de la Cognición Numérica.

Palabras clave: Trastornos Específicos del Aprendizaje, Discalculia del Desarrollo, Dislexia del Desarrollo, Cognición Numérica, niños.

Conceitua-se Cognição Numérica os padrões de ativação cerebrais que são modulados pela magnitude numérica, transmitidos pelo número; por exemplo, a quantidade "três" pode ser representada de várias maneiras: uma palavra ("três"), um dígito (3), em números romanos (III), estímulos não simbólicos (•・•), com os dedos, em uma série temporal (por exemplo, 
batendo em um tambor) ou com outras palavras (trio, triplo) que transportam a semântica, bem como o significado numérico (Cohen Kadosh et al., 2011; Cohen Kadosh \& Walsh, 2009).

Os bebês humanos, assim como outras espécies (Dehaene, 1997; Gallistel, 1990; Gallistel \& Gelman, 2000; Göbel, Shaki, \& Fischer, 2011), são sensíveis à numerosidade, estas evidências são sustentadas pelo conceito de Senso Numérico (SN) descrito por Dehaene e Cohen (1997) como uma habilidade inata para representar quantidades. Consiste, a princípio, de uma representação precisa de pequenas amostras, enquanto que grandes quantidades são compreendidas por meio de representações aproximadas (Feigenson \& Carey, 2003). O SN é uma habilidade que serve de base primária para o desenvolvimento secundário de outras habilidades, tais como processamento numérico e cálculo (Feigenson, Dehaene, \& Spelke, 2004; Iuculano, Tang, Hall, $\&$ Butterworth, 2008).

McCloskey, Caramazza e Basili (1985) com base no desempenho aritmético de indivíduos com lesões cerebrais desenvolveram um modelo para investigar a Cognição Numérica, que abrange os sistemas de Processamento Numérico e o Cálculo (CA). O processamento numérico engloba dois componentes, a Compreensão Numérica $(\mathrm{CN})$, que está associada à compreensão da natureza dos símbolos numéricos e a sua quantidade, e a Produção Numérica (PN) que envolve a escrita, leitura e contagem numérica e de objetos. Por outro lado, o sistema de CA refere-se ao tratamento de símbolos operacionais $(-,+, \mathrm{x}$ ou $\div$ ) e palavras (por exemplo, mais, menos, multiplicação), a recuperação de fatos aritméticos básicos da memória de longo prazo, e os procedimentos para executar os cálculos aritméticos (McCloskey, 1992; McCloskey et al., 1985). Deste modo, as competências para o cálculo seriam mutuamente independentes das capacidades para compreender e produzir números (Grewel, 1969); entretanto, o sistema de CA é subsidiado pelo Processamento Numérico.

Conforme o Modelo de Desenvolvimento da Cognição Numérica (von Aster \& Shalev, 2007), a transição do sistema não-simbólico (SN) para os sistemas simbólicos (verbal e visual) ocorre por meio da educação formal, e favorece o desenvolvimento de uma linha numérica mental, isto é, da habilidade de representação de valores numéricos pela associação a símbolos aprendidos, como: palavras que representam números e números arábicos, com quantidades espaciais (Geary, Hoard, Byrd-Craven, Nugent, \& Numtee, 2007), ao longo dos anos, inclusive durante a idade adulta. No entanto, estas transições dependem de habilidades matemáticas intactas, estimuladas pela experiência, plasticidade, desenvolvimento de outras habilidades cognitivas, assim como por fatores socioculturais e educacionais, os quais podem influenciar tanto o processamento numérico quanto o sistema de CA (Dellatolas, von Aster, Braga, Meier, \& Deloche, 2000, Gross-Tsur, Manor, \& Shalev, 1996; Hein, Bzufka, \& Neumärker, 2000; Koumoula et al., 2004; Santos et al., 2012b).

Com o intuito de compreender como os fatores de desenvolvimento, idade, meio ambiente e gênero influenciam no desenvolvimento das habilidades matemáticas, Santos et al. (2012b) avaliaram a Cognição Numérica de crianças brasileiras de 7 a 12 anos de idade, de escolas públicas, utilizando a Zareki-R (Batterie pour l'évaluation du traitement des nombres et $d u$ calcul chez l'enfant; von Aster \& Dellatolas, 2006). Participaram do estudo 172 crianças divididas em dois grupos: urbanas $(N=119)$ e rurais $(N=53)$. Crianças da zona rural apresentaram escores inferiores em apenas um aspecto do processamento numérico - a comparação escrita de quantidades e numa medida de $\mathrm{CN}$ - portanto, existiria uma baixa influência dos aspectos ambientais determinando o desempenho. Crianças de 7 a 8 anos de idade demonstraram uma pontuação inferior global quando comparadas as crianças com mais idade, confirmando que o desempenho da maioria das habilidades matemáticas está relacionado à idade, além disso, meninos apresentaram um desempenho superior em processamento numérico e CA. Os autores concluíram que a Zareki-R é um instrumento adequado para identificar o desenvolvimento das habilidades matemáticas, que é influenciada por 
fatores como o ambiente, idade e sexo. Este foi o primeiro estudo brasileiro utilizando a Zareki-R e demonstrou também a validade de constructo da bateria em relação ao Teste de Desempenho Escolar (Stein, 1994).

Considerando a complexidade dos sistemas relacionados à Cognição Numérica, é necessário avaliar vários campos numéricos, como $\mathrm{SN}$, $\mathrm{CN}, \mathrm{PN}$ e $\mathrm{CA}$, para promover um diagnóstico diferencial e estabelecer estratégias adequadas de intervenção (Moll, Göbel, \& Snowling, 2014; von Aster, 2000). Santos, Silva, Ribeiro, Dellatolas, e von Aster (2012a) investigaram os sistemas da Cognição Numérica em crianças com desenvolvimento típico e verificaram que crianças brasileiras de $1^{\mathrm{a}}$ e $2^{\mathrm{a}}$ Série, atuais $2^{\circ}$ e $3^{\circ}$ Ano, respectivamente, possuem uma desempenho global inferior para os constructos de $\mathrm{CN}$, PN e CA, o que não ocorre para SN. Além disso, não foram encontradas diferenças entre meninos e meninas. Por fim, esses resultados demonstraram que a realização da Zareki-R é pouco influenciada pelo SN em comparação com os outros três sistemas, que são dependentes do ensino formal (Santos et al., 2012a), sendo de interesse compreender como tais sistemas de Cognição Numérica funcionam em crianças com Transtornos Específicos de Aprendizagem por meio da bateria.

Os Transtornos Específicos de Aprendizagem relacionam-se às disfunções do desenvolvimento neurocognitivo envolvidas no ato de aprender (Butterworth \& Kovas, 2013); diferem da dificuldade de aprendizagem - que é extrínseca ao indivíduo e decorrente de falhas pedagógicas e/ou fatores socioculturais - e demandam tratamento especializado para suas manifestações, sendo as mais comuns: a Dislexia do Desenvolvimento (DL), a Discalculia do Desenvolvimento (DD) e a Disgrafia do Desenvolvimento, as quais podem ocorrer isoladamente ou combinadas entre si, todas estas limitações devem ser investigadas. Quando são satisfeitos os critérios para mais de um Transtorno Específico da Aprendizagem (American Psychiatric Association [APA], 2013), neste último caso constitui-se o Transtorno Misto de Habilidades Escolares (F81.3; Organização Mundial da Saú- de [OMS], 1993), como é o caso da Discalculia do Desenvolvimento combinada com dislexia (DDc). Por fim, são condições intrínsecas ao indivíduo (Ciasca, 2003) e que, podem, eventualmente, ocorrer em comorbidade com outros Transtornos Mentais e do Comportamento que ocorrem na infância e adolescência (F.80-F.98, CID-10; OMS, 1993).

Neste artigo serão tratadas, de maneira específica, a DD e DL, a Tabela 1 apresenta as respectivas descrições.

O termo Discalculia do Desenvolvimento (DD) foi introduzido por Ladislav Kosc (1974). A DD se caracteriza como um transtorno que afeta a aquisição normal das habilidades aritméticas (Butterworth, Varma, \& Laurillard, 2011; Geary, 2010; Gross-Tsur et al., 1996; Shalev, 2004; von Aster et al., 2007; von Aster \& Shalev, 2007).

Manifesta-se nos primeiros anos escolares por dificuldades na recuperação da aritmética básica e em exercícios de computação aritmética (Geary, 1995), ou seja, em tarefas simples de processamento numérico, como comparar e nomear dígitos, contar em sequência e contar pequenos números de pontos, obtendo médias um, dois ou mais desvios padrões abaixo do esperado em comparação às crianças de mesma escolaridade (Landerl, Bevan, \& Butterworth, 2004; Landerl, Fussenegger, Moll, \& Willburger, 2009; Landerl \& Kölle, 2009; Mejias, Gregoire, \& Noel, 2012; Rousselle \& Noël, 2008; Skagerlund \& Träff, 2014). Rotzer et al. (2009) acrescentaram que o diagnóstico de DD poderia ser tanto pelo prejuízo em três subtestes como no escore total de uma bateria especializada, de pelo menos um desvio-padrão e meio, abaixo do esperado para a idade.

Ashkenazi, Mark-Zigdon e Henik (2009) constataram que crianças com DD apresentam um número maior de erros na comparação numérica entre números de um dígito e maior tempo de reação na comparação de números de dois dígitos em relação a crianças do grupo controle. Contudo, podem adquirir conceitos básicos e escrever números, ler, ou relacioná-los às palavras correspondentes (Gross-Tsur et al., 1996; Shalev, Manor, Amir, \& Gross-Tsur, 1993). 
Tabela 1

Descrição da Discalculia do Desenvolvimento e da Dislexia do Desenvolvimento

\begin{tabular}{ll}
\hline & \multicolumn{1}{c}{ Discalculia do Desenvolvimento } \\
\hline Taxonomia & $\begin{array}{l}\text { 'Transtornos específicos de aprendizagem } \\
\text { com prejuízos na matemática ou Discalculia } \\
\text { como termo alternativo' (315.1, DSM-V; APA, } \\
\text { 2013) e 'Transtorno específico de habilidades } \\
\text { aritméticas' (F81.2, CID-10; OMS, 1993). }\end{array}$ \\
Caracterização & Um tTranstorno neurológico de origem biológica \\
Clínica & que se manifesta na dificuldade de aprendizagem \\
& e problemas na aquisição de habilidades \\
& acadêmicas acentuadamente abaixo do nível da \\
& idade e se manifesta nos primeiros anos escolares, \\
& com duração de pelo menos 6 meses; não \\
& atribuído à deficiência intelectual, transtornos do \\
& desenvolvimento, ou neurológica ou distúrbios \\
& motores com prejuízos específicos no Senso \\
& numérico, Memorização de fatos aritméticos, \\
& Cálculo preciso e raciocínio matemático exato \\
& (APA, 2013).
\end{tabular}

Transtorno que implica uma alteração específica da habilidade em aritmética, não atribuível exclusivamente a um retardo mental global ou à escolarização inadequada. O déficit concerne ao domínio de habilidades computacionais básicas de adição, subtração, multiplicação e divisão mais do que as habilidades matemáticas abstratas envolvidas na álgebra, trigonometria, geometria ou cálculo (OMS, 1993).

Epidemiologia Está presente entre 3 a $6 \%$ da população escolar, contudo, apenas $1,8 \%$ apresentam a manifestação pura da DD enquanto $4.2 \%$ apresentam comorbidade com DL (DD combinada com DL [DDc]; von Aster, Schweiter, \& Weinhold Zulauf, 2007; von Aster \& Shalev, 2007).

Genética A probabilidade de ocorrência da DD em famílias que possuem o genótipo para esta é dez vezes maior do que na população em geral, assim como sua probabilidade entre gêmeos monozigóticos é maior do que entre os dizigóticos (Price, Holloway, Räsänen, Vesterinen, \& Ansari, 2007).
'Transtornos específicos de aprendizagem com prejuízos na leitura ou dislexia como termo alternativo' (315.00, DSM-V; APA, 2013) e Transtorno específico de Leitura (F81.0, CID-10; OMS, 1993).

Um transtorno neurológico de origem biológica que se manifesta na dificuldade de aprendizagem e problemas na aquisição de habilidades acadêmicas acentuadamente abaixo do nível da idade e se manifesta nos primeiros anos escolares, com duração de pelo menos 6 meses; não atribuído à deficiência intelectual, transtornos do desenvolvimento, ou neurológica ou distúrbios motores com prejuízos específicos na precisão de leitura de palavras, fluência e compreensão na leitura (APA, 2013)

A característica essencial é um comprometimento específico e significativo do desenvolvimento das habilidades da leitura, não atribuível exclusivamente à idade mental, a transtornos de acuidade visual ou escolarização inadequada. A capacidade de compreensão da leitura, o reconhecimento das palavras, a leitura oral, e o desempenho de tarefas que necessitam da leitura podem estar todas comprometidas. O transtorno específico da leitura se acompanha frequentemente de dificuldades de soletração, persistindo comumente na adolescência, mesmo quando a criança haja feito alguns progressos na leitura. As crianças que apresentam um transtorno específico da leitura têm frequentemente antecedentes de transtornos da fala ou de linguagem. $O$ transtorno se acompanha comumente de transtorno emocional e de transtorno do comportamento durante a escolarização (OMS, 1993).

A prevalência oscila entre 3 a $10 \%$ na população escolar (Karande, 2005).

A probabilidade de ocorrência de DL em famílias que possuem o genótipo para esta, quando relacionada a leitura de palavras, situa-se entre $50 \%$ e $60 \%$, e quanto a disgrafia entre $50 \%$ e $70 \%$ (Schulte-Körne, Warnke, \& Remschmidt, 2006). 
Substrato As crianças com DD apresentam diferenças na Neuroanatômico ativação de regiões parietais do encéfalo, mais especificadamente sulco intraparietal (IPS). A DD tem como base a hipoperfusão, ou seja, redução do fluxo sanguíneo, tanto no sulco intraparietal esquerdo quanto no direito (Cohen Kadosh et al., 2011; Cohen Kadosh \& Walsh, 2009).
Diversos estudos demonstraram uma hipoperfusão em crianças com DL nas regiões parieto-temporal e occipitotemporal esquerdo. Muitos estudos também relataram hiperperfusão nas áreas inferiores, esquerda e direita dos giros frontais (Hoeft et al., 2011).

Observações Não atribuíveis exclusivamente à idade mental, a transtornos de acuidade visual ou escolarização inadequada (OMS, 1993). Em presença de um déficit sensorial, as dificuldades na capacidade excedem aquelas geralmente a estes associadas (APA, 2013).

Silva e Santos (2011) investigaram quais os aspectos da Cognição Numérica e da memória operacional estavam relacionados a déficits em aritmética em crianças brasileiras de 9 a 10 anos de idade com Transtornos Específicos de Aprendizagem, divididas em dois grupos com base na pontuação de cálculo: com dificuldade em aritmética $(N=19)$ e sem dificuldades em aritmética $(N=11)$. As crianças com dificuldade em aritmética apresentaram resultados ligeiramente mais baixos do que o outro grupo em relação ao nível intelectual e memória operacional visuoespacial, bem como déficits nos subtestes da Zareki-R: ditado de números $(\mathrm{PN})$, cálculo mental e resolução de problemas (CA). Houve correlações moderadas entre o cálculo mental e medidas de memória operacional (fonológica e visuoespaciais). $\mathrm{O}$ desempenho das crianças com dificuldade em aritmética na Zareki-R foi maior que um desvio padrão e meio abaixo dos dados normativos, o que condiz com os critérios para diagnóstico da DDc adotados por Rotzer et al. (2009).

Os autores também realizaram uma análise qualitativa do desempenho das crianças com DDc no subteste Problemas Aritméticos da Zareki-R e verificaram que mais de um quinto das crianças apresentaram erros na escolha do procedimento (Silva \& Santos, 2011), o que corrobora falhas no processamento dos símbolos ou palavras operacionais e na execução de procedimentos de cálculos aritméticos (McCloskey et al., 1985). Além disso, mais de $70 \%$ das crianças com DDc foram incapazes de responder pelo menos um dos seis itens do subteste, confirmando um atraso significativo na conso- lidação dos sistemas numéricos simbólicos e na aquisição do pensamento aritmético (Mundy \& Gilmore, 2009; von Aster \& Shalev, 2007), não houve evidência de que tais erros decorressem predominantemente de déficits em produção numérica.

Quanto à Dislexia do Desenvolvimento, esta é caracterizada pelo prejuízo em leitura de palavras simples, que esta relacionada a déficits na decodificação fonológica e soletração que acomete indivíduos sem deficiências sensoriais ou experiências educacionais inadequadas (Snowling \& Stackhouse, 2004). Prejuízos secundários costumam ser problemas na compreensão de textos e, consequentemente, desenvolvimento reduzido de vocabulário e de conhecimentos gerais (Oliveira, Sacchetto, Ueki, Silva, \& Macedo, 2011); além do prejuízo no processamento fonológico também estão comprometidos elementos subjacentes a leitura e escrita, como a atenção, velocidade de leitura e estratégias de interação com o texto, que são inadequadas (Capovilla, Gutschow, \& Capovilla, 2004; Machado \& Capellini, 2011; Mousinho, Correa, \& Mesquita, 2010; Salgado, 2005). Quanto à escrita, as crianças com DL costumam apresentar dificuldades mais extensas do que em leitura, pois além da complexidade cognitiva imposta pela própria escrita, o processo de conversão fonológico-ortográfico, separadamente, é insuficiente para a obtenção da correta ortografia de palavras (Dias \& Ávila, 2008). Um estudo realizado por Freitas, Ferreira e Haase (2010) encontrou correlações entre as habilidades cognitivas linguísticas e aritméticas, mais especifi- 
cadamente para habilidade relacionada ao cálculo. Rubinsten e Henik (2006) demonstraram dissociações entre adultos com DL e DD; em que o grupo com DD possuía problemas para associar automaticamente algarismos arábicos com a sua representação interna de magnitudes, mas não tinha dificuldades em associar automaticamente letras com seus fonemas, enquanto que o grupo DL demonstrou o padrão oposto; em outras palavras, os estudos demonstram que crianças com DL tendem a desempenhar bem tarefas matemáticas, com dificuldades eventuais secundárias às suas limitações em leitura e escrita (Steeves, 1983).

O presente estudo investigou o desenvolvimento dos sistemas da Cognição Numérica ( $\mathrm{SN}$, $\mathrm{CN}$, PN e CA) na referida amostra de crianças brasileiras com Transtornos Específicos de Aprendizagem (Silva \& Santos, 2011), considerando a influência da escolaridade. A hipótese é de que o grupo de crianças com perfil de DL não apresentará prejuízos significativos nos diferentes sistemas da Cognição Numérica, visto que os seus prejuízos geralmente são relacionados à leitura e à escrita per se (Landerl et al., 2004). Por outro lado, espera-se que crianças com perfil de DDc apresentarão preservação do SN (von Aster \& Shalev, 2007), contudo, déficits significativos nos outros sistemas da Cognição Numérica, principalmente em CA. Além disso, há a hipótese que esses déficits entre as crianças com DDc sejam independentes da escolaridade, visto que os Transtornos Específicos de Aprendizagem não são decorrentes de fatores pedagógicos e/ou fatores socioculturais.

\section{Método}

\section{Participantes}

Participaram do estudo 72 crianças (36 meninos), de idade entre 9 e 10 anos, matriculadas em escolas públicas da região Centro Oeste Paulista, dos $4^{\circ}$ e $5^{\circ}$ anos do Ensino Fundamental. As crianças foram divididas em três grupos: controle (CT, $N=42$ ), com perfil de Dislexia do Desenvolvimento (DL, $N=11$ ) e com perfil de Discalculia do Desenvolvimento combinada com dislexia (DDc, $N=19$ ), balanceados por sexo e idade. As crianças CT apresentaram nível intelectual normal - QIV $=106,26 \pm 13,14$ (WISC-III; Wechsler, 2002), assim como as crianças DL - percentil $=61,72 \pm 19,20-$ e DDc - percentil $=45,26 \pm 11,72$ (Angelini, Alves, Custódio, Duarte, \& Duarte, 1999) contudo, as crianças destes dois últimos grupos frequentavam salas de reforço escolar em decorrência de Transtornos Específicos de Aprendizagem evidenciados pelo Teste de Desempenho Escolar ( $4^{\circ}$ Ano, Escore Bruto Total - ETB $=80,56 \pm$ 24,46; $5^{\circ}$ Ano, ETB = 88,56 \pm 17,49; Stein, 1994), no qual a média do desempenho das crianças foi correspondente ao de crianças de dois anos escolares abaixo do que estavam matriculadas, isto é, $2^{\circ}$ e $3^{\circ}$ Ano respectivamente. Além disso, como critério específico, as crianças com DDc apresentavam necessariamente classificação 'inferior' no Teste de Aritmética do TDE. Os critérios de exclusão foram evidências de desordens neurológicas, psiquiátricas ou de outra ordem, subnutrição, reprovação escolar, distúrbios de comportamento ou uso de substâncias psicoativas aferidos na ocasião da anamnese. Não houve diferença significativa entre os grupos quanto ao nível socioeconômico $[F(2,66)=1,54 ; p=0,22]$ e os três grupos obtiveram classificação $\mathrm{C}$ ou média (35-58 pontos, Associação Brasileira dos Institutos de Pesquisa de Mercado [ABIPEME]; Almeida \& Wickerhauser, 1991).

O estudo foi aprovado pelo Comitê de Ética da Universidade Estadual Paulista "Júlio de Mesquita Filho", processos no. 1637/2005 e no. $355 / 2005$. Posteriormente, foi realizado contato com as escolas da rede pública de ensino seguida de apresentação coletiva do projeto aos pais. Após a assinatura do Termo de Consentimento Livre e Esclarecido, os pais realizaram uma entrevista de anamnese para ser contrastada com os critérios de exclusão. Na etapa seguinte realizou-se a avaliação neurocognitiva, individualmente com cada criança, em sala com boas condições físicas das próprias escolas. Os testes foram administrados em ordem aleatória entre outros instrumentos cognitivos e foi realizado em uma sessão única de aproximadamente $1 \mathrm{~h} 40$ minutos, com intervalo de 20 minutos para evitar efeito de fadiga. 


\section{Cognição Numérica}

A Bateria de Testes Neuropsicológicos para Processamento e Cálculo em Crianças - Revisada, ou Zareki-R (von Aster \& Dellatolas, 2006) é uma bateria de testes internacional especializada para avaliar a Cognição Numérica de crianças em idade escolar. Dados normativos com pontuações para crianças brasileiras foram apresentados por Santos et al. (2012b). O Zareki-R tem 11 subtestes especializados em habilidades matemáticas e um subteste de memória operacional fonológica (Memorização de Dígitos). A pontuação total é composta pela soma dos subtestes, exceto Memorização de Dígitos. Para o objetivo desse artigo, os subtestes foram divididos em quatro construtos ou sistemas: Senso Numérico (SN), composto pela soma de pontos de Enumeração de Pontos e Estimativa Visual de Quantidades; Compreensão Numérica $(\mathrm{CN})$, composto pela soma de Comparação de Números Apresentados Oralmente, Comparação de Números Escritos e Estimativa Qualitativa de Quantidades no Contexto; Produção Numérica (PN), composto pela soma de Contagem Oral em Ordem Inversa, Ditado de Números e Leitura de Números; Cálculo (CA), composto pela soma de Cálculo Mental e Problemas Aritméticos Apresentados Oralmente. O subteste Posicionamento de números em escala vertical não foi incluído nas pontuações, porque se tratar de uma medida relacionada a linha numérica mental, que sozinho não poderia ser definido como escore composto. A descrição de cada subteste foi apresentada em Santos et al. (2012b) e Silva e Santos (2011).

\section{Análise Estatística}

Os dados obtidos foram analisados utilizando o software do STATISTICA - versão 7, Statsoft Incorporation 1984-2004 (StatSoft, 2004). As análises com propostas inferenciais foram realizadas em duas etapas. Na primeira, a análise de variância (ANOVA) foi realizada para verificar efeito de grupo em relação aos quatro sistemas da Cognição Numérica Zareki-R. Neste caso, a variável independente adotada foram os 3 grupos (CT, DL e DDc) e as variáveis de- pendentes foram os escores nos 4 sistemas da Zareki-R (SN, CN, PN e CA). Na segunda etapa, pelo fato de ter sido observada anteriormente relações entre desempenho e idade e escolaridade na Zareki-R (Santos et al., 2012a, 2012b), foi realizada uma análise de covariância (ANCOVA), escolaridade como covariável, em contraste com as mesmas variáveis dependentes da primeira análise. Para todas as análises, o teste post-hoc de Tukey para amostras desiguais foi adotado com nível alpha de significância de $p$ $\leq 0,05$.

\section{Resultados}

As análises por meio da ANOVA revelaram efeito de grupo para três dos quatro siste$\operatorname{mas}[F(8,132)=6,00 ; p<0,0001]$, exceto $\mathrm{SN}$. $\mathrm{O}$ desempenho do grupo $\mathrm{CT}$ foi superior ao do grupo DDc no sistema $\mathrm{CN}$, enquanto tanto o grupo CT quanto o DL tiveram escores superiores ao do grupo DDc nos sistemas $\mathrm{PN}$ e CA. Vide Tabela 2.

Uma vez que as crianças pertenciam a duas séries escolares, este fator poderia ter determinado a diferença entre os grupos, por este motivo uma ANCOVA, tendo a escolaridade como covariável, foi conduzida sendo que o efeito de grupo permaneceu $[F(8,130)=6,13 ; p<$ 0,0001]. Vide Tabela 2.

Devido ao número restrito de crianças em cada grupo, análises exploratórias utilizando o teste não paramétrico de Kruskal-Wallis foram conduzidas para controlar o efeito das múltiplas comparações, as quais confirmaram os resultados obtidos: Senso Numérico $[\chi 2(2,72)=1,53$; $p=0,46]$, Compreensão Numérica $[\chi 2(2,72)=$ 9,36; $p<0,01]$, Produção Numérica $[\chi 2(2,72)$ $=10,56 ; p<0,01]$ e Cálculo $[\chi 2(2,72)=16,59$; $p<0,01]$.

\section{Discussão}

O objetivo deste estudo foi investigar o desenvolvimento dos sistemas específicos da Cognição Numérica ( $\mathrm{SN}, \mathrm{CN}, \mathrm{PN}$ e CA) em crianças brasileiras com Transtornos Específicos de Aprendizagem, sendo um grupo com perfil de 
Tabela 2

Desempenho [M(DP)] na Zareki-R, por Grupos e Nível Escolar

\begin{tabular}{|c|c|c|c|c|c|}
\hline & $N$ & SN & $\mathrm{CN}$ & PN & $\mathrm{CA}$ \\
\hline & & $M(D P)$ & $M(D P)$ & $M(D P)$ & $M(D P)$ \\
\hline \multicolumn{6}{|l|}{ Grupos } \\
\hline CT & 42 & $10,14(2,60)$ & $47,10(5,40)$ & $33,64(3,05)$ & $40,90(9,42)$ \\
\hline DL & 11 & $10,00(2,41)$ & $41,64(6,56)$ & $31,45(4,08)$ & $39,82(6,45)$ \\
\hline DDc & 19 & $9,42(2,14)$ & $40,16(7,20)$ & $26,74(7,40)$ & $23,00(13,02)$ \\
\hline \multicolumn{6}{|c|}{ Escolaridade } \\
\hline \multicolumn{6}{|l|}{$4^{\circ}$ Ano } \\
\hline $\mathrm{CT}$ & 17 & $9,94(2,51)$ & $47,41(5,51)$ & $33,71(1,72)$ & $38,41(10,07)$ \\
\hline $\mathrm{DL}$ & 7 & $9,00(2,24)$ & $42,29(5,47)$ & $31,29(4,50)$ & $40,43(7,04)$ \\
\hline DDc & 9 & $8,67(2,00)$ & $37,78(6,12)$ & $22,22(7,90)$ & $17,89(11,85)$ \\
\hline \multicolumn{6}{|l|}{$5^{\circ}$ Ano } \\
\hline $\mathrm{CT}$ & 25 & $10,28(2,70)$ & $46,88(5,42)$ & $33,60(3,73)$ & $42,60(8,76)$ \\
\hline DL & 4 & $11,75(1,71)$ & $40,50(9,00)$ & $31,75(3,86)$ & $38,75(6,08)$ \\
\hline DDc & 10 & $10,10(2,13)$ & $42,30(7,72)$ & $30,80(3,91)$ & $27,60(12,83)$ \\
\hline
\end{tabular}

Notas. Legenda: $N=$ número de participantes; $\mathrm{SN}=$ Senso Numérico; $\mathrm{CN}=$ Compreensão Numérica; $\mathrm{CA}=\mathrm{Cálculo} ; M=$ média; $D P=$ desvio-padrão; $\mathrm{CT}=$ controles; $\mathrm{DL}=$ perfil de Dislexia do Desenvolvimento; $\mathrm{DDc}=$ perfil de Discalculia do Desenvolvimento combinada com dislexia.

Dislexia do Desenvolvimento (DL) e outro perfil de Discalculia do Desenvolvimento combinada com dislexia (DDc).

Conforme observado nas análises, o Senso Numérico não se mostrou prejudicado para nenhum dos grupos com transtornos específicos de aprendizagem. Era esperada a preservação do SN em crianças com DL, pois as habilidades básicas em matemática são inatas e anteriores à aquisição da linguagem, embora se ampliem a partir dela (Landerl et al., 2004; Tressoldi, Rosatib, \& Lucangelic, 2007). Nos casos de DD pura há evidências de que o SN esteja prejudicado (Landerl et al., 2009; Landerl \& Kölle, 2009; Mejias et al., 2012), enquanto em crianças com DDc este é um aspecto geralmente preservado (von Aster \& Shalev, 2007).

Em relação ao sistema de Compreensão Numérica, pode-se observar que do ponto de vista estatístico as crianças DDc apresentaram diferenças significativas apenas em relação às crianças do grupo controle; corroborando estudos prévios (Landerl et al., 2009; Landerl \& Kölle, 2009; Mejias et al., 2012; Rousselle \& Noël, 2008). Entretanto, a apreciação clínica dos escores revela que qualitativamente, tanto o desempenho das crianças DDc quanto DL estava levemente prejudicado (1 DP abaixo da média), sendo que para este último grupo, o desempenho estava no limiar para um desempenho médio. De acordo com a literatura, os déficits de crianças DL são mais relacionados ao código verbal/fonológico de magnitudes numéricas, por exemplo, em tarefas de comparação oral de quantidades (Ashkenazi et al., 2009; Landerl et al., 2004; Tressoldi et al., 2007). No presente estudo, ao considerar o sistema $\mathrm{CN}$ de maneira ampla, isto é, por meio de um escore composto pela soma de 
diferentes subtestes, as crianças DL exibiram um desempenho geral normal, contudo, ao contrário da hipótese inicial, considerando os valores clínicos de referência, houve um prejuízo leve na decodificação de diferentes símbolos numéricos, possivelmente mais relacionados às limitações próprias de suas características clínicas (Landerl et al., 2004; Landerl et al., 2009).

Nos sistemas de Produção Numérica e Cálculo as crianças com DL apresentaram desempenho equivalente ao de crianças com desenvolvimento típico e superior ao grupo DDc, discrepância entre grupos que corrobora com os Rubinsten e Henik (2006), e constitui o principal achado do presente estudo, pois, permite a distinção clara entre os dois grupos, estando em favor de distinções nosográficas. Enquanto as crianças DL exibiram déficits restritos à Compreensão Numérica, as crianças DDc apresentaram prejuízos moderados (2 DP abaixo do esperado) em Produção Numérica e de leve a moderado $(1,5$ $D P$ abaixo do esperado) em Cálculo. Santos et al. (2012a, 2012b) observaram em crianças de 7 e 8 anos etários com desenvolvimento típico dificuldades em tarefas de Produção Numérica. No presente estudo, as crianças com DDc possuíam entre 9 e 10 anos de idade e, apesar disso, exibiram uma pontuação correspondente ao de crianças dos primeiros anos escolares denotando um atraso significativo na aquisição desse sistema; sendo esta informação expressiva do ponto de vista diagnóstico. Este resultado confirma achados anteriores, de que crianças com DD pura ou DDc apresentam prejuízos em transcodificação numérica, isto é, ditado e/ou leitura de números (Rotzer et al., 2009; Shalev, 2004; Silva \& Santos, 2011; Temple, 1989; Zuber, Pixner, Moeller, \& Nuerk, 2009), demandando um maior tempo de reação na execução desta tarefa, apesar de haver precisão na resposta (Rubinsten \& Henik, 2006). Convém ressaltar que os prejuízos observados em Produção Numérica de nossa amostra foram mais significativos do que em Cálculo, sendo, portanto, fundamental o desenvolvimento de estratégias de reabilitação neurocognitiva focadas na escrita, leitura e contagem numérica e de objetos.
Quanto ao sistema Cálculo, especificamente, o desempenho médio das crianças DDc foi de leve a moderadamente prejudicado $(1,5 \mathrm{DP}$ abaixo da média dos controles). Em geral, o desempenho em cálculo exige aspectos de processamento numérico (CN e PN; McCloskey et al., 1985), sendo que o prejuízo aritmético em crianças com DDc são relacionados tanto a prejuízos específicos em Cálculo quanto aos déficits em Compreensão e Produção Numérica, de maneira interdependente. Silva e Santos (2011) observaram, contudo, erros relacionados especificamente ao cálculo mental (escolha do procedimento adequado) e a uma ineficiência em consolidar conhecimentos aritméticos, visto a alta porcentagem de respostas ao acaso e ausência de tentativas de responder aos cálculos.

Conforme a análise de covariância, as diferenças quanto aos sistemas de Cognição Numérica não foram influenciadas pela escolaridade, corroborando com a hipótese inicial de mesmo teor, o que se justifica pelo fato de os Transtornos Específicos de Aprendizagem serem intrínsecos aos sujeitos (Ciasca, 2003). Contudo, ficou evidente que habilidades matemáticas em crianças DDc estavam defasadas em relação às crianças DL e àquelas do grupo controle. Conforme observam Santos et al. (2012b), as crianças brasileiras com desenvolvimento típico apresentam um nivelamento no desempenho matemático a partir do terceiro ano de escolaridade $(M=9,02$ $\pm 0,17$ anos), inclusive aferido pela Zareki-R, significativamente superior e qualitativamente diferente em relação ao das crianças DDc, o que confirma a nossa hipótese inicial de discrepância quanto ao conhecimento aritmético.

Esse baixo desempenho das crianças com DDc em Cognição Numérica têm sido justificado na literatura não por prejuízos em habilidades matemáticas básicas (em SN; von Aster \& Shalev, 2007), mas por um empobrecimento do processamento e da compreensão de símbolos numéricos (Iuculano et al., 2008; Landerl \& Kölle, 2009). Em parte, esse empobrecimento tem sido relacionado a uma falta de automatização do conhecimento matemático (fatos numéricos) adquirido durante a aprendizagem, levando a uma 
lentidão no desempenho que não seria correspondente ao esperado (Landerl et al., 2009; Landerl \& Kölle, 2009; Mejias et al., 2012; Rousselle \& Nöel, 2008; Skagerlund \& Träff, 2014).

Uma limitação deste estudo foi que o número de participantes por grupos era pequeno e desbalanceado, principalmente entre aqueles com Transtornos Específicos de Aprendizagem. Contudo, a seleção dos participantes foi criteriosa, o que preveniu a influência de variáveis que poderiam confundir as interpretações apresentadas. Como exemplo, pode-se citar a escolha por crianças sem repetição escolar em todos os grupos; dessa forma as crianças com Transtornos Específicos de Aprendizagem frequentavam as mesmas escolas, eram submetidas à mesma forma de ensino e estudavam em salas equivalentes as das crianças com desenvolvimento típico, o que favorece a comparação entre elas quanto ao nível de conhecimento e competência matemática, propiciando credibilidade aos resultados observados.

Como conclusão, mesmo as crianças com transtornos específicos de aprendizagem em diferentes habilidades apresentaram preservação em SN, reforçando o seu caráter inato. Tanto as crianças DL quanto DDc exibiram prejuízos leves em Compreensão Numérica. De maneira particular, as crianças DDc apresentaram déficits significativos em Produção Numérica e Cálculo, ou seja, seus prejuízos em Cognição Numérica foram mais generalizados. Portanto, a Zareki-R permitiu identificar peculiaridades no que diz respeito aos sistemas específicos da Cognição Numérica em que as crianças DDc apresentaram prejuízos quantitativos e qualitativamente maiores e defasagem em relação às crianças DL e com desenvolvimento típico.

\section{Referências}

Almeida, P. M., \& Wickerhauser, H. (1991, abr./ jun.). O critério ABA/ABIPEME - Em busca de uma atualização. Um estudo e uma proposta submetidos à ABA e à ABIPEME. Manuscrito de circulação restrita da Associação Brasileira de Anunciantes e Associação Brasileira dos Institutos de Pesquisa de Mercado, São Paulo, SP, Brasil.
American Psychiatric Association. (2013). Diagnostic and statistical manual of mental disorders ( $5^{\text {th }}$ ed.). Arlington, VA: Author.

Angelini, A. L., Alves, I. C. B., Custódio, E. M., Duarte, W. F., \& Duarte, J. L. M. (1999). Manual: Matrizes Progressivas Coloridas de Raven. São Paulo, SP: Centro Editor de Testes e Pesquisas em Psicologia.

Ashkenazi, S., Mark-Zigdon, N., \& Henik, A. (2009). Numerical distance effect in developmental dyscalculia. Cognitive Development, 24, 387-400. doi:10.1016/j.cogdev.2009.09.006

Butterworth, B., \& Kovas, Y. (2013). Understanding neurocognitive developmental disorders can improve education for all. Science, 340, 300-305. doi:10.1126/science. 1231022

Butterworth, B., Varma, S., \& Laurillard, D. (2011). Dyscalculia: From brain to education. Science, 332, 1049-1053. doi:10.1126/science. 1201536

Capovilla, A. G. S., Gutschow, C. R. D., \& Capovilla, F. C. (2004). Habilidades cognitivas que predizem competência de leitura e escrita. Psicologia: Teoria e Prática, 6, 13-26.

Ciasca, S. M. (2003). Distúrbios e dificuldades de aprendizagem: Questão de nomenclatura. In S. M. Ciasca, Distúrbios de aprendizagem: Proposta de avaliação interdisciplinar (pp. 19-32). São Paulo, SP: Casa do Psicólogo.

Cohen Kadosh, R., Bahrami, B., Walsh, V., Butterworth, B., Popescu, T., \& Price, C. J. (2011). Specialization in the human brain: The case of numbers. Frontiers in Human Neuroscience, 5, 1-9. doi:10.3389/fnhum.2011.00062

Cohen Kadosh, R., \& Walsh, V. (2009). Numerical representation in the parietal lobes: Abstract or not abstract? Behavioral and Brain Sciences, 32, 313-328. doi:10.1017/S0140525X09990938

Dehaene, S. (1997). The number sense: How the mind creates mathematics. New York: Oxford University Press.

Dehaene, S., \& Cohen, L. (1997). Cerebral pathways for calculation: Double dissociation between rote verbal and quantities knowledge of arithmetic. Cortex, 33, 219-250. doi:10.1016/S00109452(08)70002-9

Dellatolas, G., von Aster, M., Braga, L. W., Meier, M., \& Deloche, G. (2000). Number processing and mental calculation in school children aged 7 to 10 years: A transcultural comparison. Euro- 
pean Child and Adolescent Psychiatry, 9(Suppl. 2), 102-110. doi:10.1007/s007870070003

Dias, R. S., \& Ávila, C. R. B. (2008). Uso e conhecimento ortográfico no transtorno específico de leitura. Revista da Sociedade Brasileira de Fonoaudiologia, 13, 381-390. doi:10.1590/S151680342008000400014

Feigenson, L., \& Carey, S. (2003). Tracking individuals via object-files: Evidence from infants' manual search. Developmental Science, 6, 568584. doi:10.1111/1467-7687.00313

Feigenson, L., Dehaene, S., \& Spelke, E. S. (2004). Core systems of number. Trends in Cognitive Sciences, 8, 307-314. doi:10.1016/j. tics.2004.05.002

Freitas, N. L., Ferreira, F. O., \& Haase, V. G. (2010). Linguagem e matemática: Estudo sobre relações entre habilidades cognitivas linguísticas e aritméticas. Ciências \& Cognição, 15, 111-125.

Gallistel, C. R. (1990). The organization of learning. Cambridge, MA: Massachusetts Institute of Technology Press.

Gallistel, C. R., \& Gelman, R. (2000). Non-verbal numerical cognition: From reals to integers. Trends in Cognitive Sciences, 4, 59-65. doi:10.1016/ S1364-6613(99)01424-2

Geary, D. C. (1995). Reflections of evolution and culture in children's cognition: Implications for mathematical development and instruction. American Psychologist, 50, 24-37. doi:10.1037/0003-066X.50.1.24

Geary, D. C. (2010). Mathematical learning disabilities. In J. Holmes (Ed.), Advances in child development and behavior (Vol. 38, pp. 45-77). San Diego, CA: Academic Press.

Geary, D. C., Hoard, M. K., Byrd-Craven, J., Nugent, L., \& Numtee, C. (2007). Cognitive mechanisms underlying achievement deficits in children with mathematical learning disability. Child Development, 78, 1343-1359. doi:10.1111/j.14678624.2007.01069.x

Göbel, S. M., Shaki, S., \& Fischer, M. H. (2011). The Cultural Number Line: A review of cultural and linguistic influences on the development of number processing. Journal of Cross-Cultural Psychology, 42, 543-565. doi:10.1177/0022022111406251

Grewel, F. (1969). The acalculias. In P. J. Vinken \& G. W. Bruyn (Eds.), Handbook of clinical neurology (Vol. 4, pp. 181-196). New York: Wiley.
Gross-Tsur, V., Manor, O., \& Shalev, R. S. (1996). Developmental dyscalculia: Prevalence and demographic features. Developmental $\mathrm{Me}$ dicine and Child Neurology, 38, 25-33. doi:10.1111/j.1469-8749.1996.tb15029.x

Hein, J., Bzufka, M. W., \& Neumärker, K. J. (2000). The specific disorder of arithmetic skills. Prevalence studies in a rural and an urban population sample and their clinico-neuropsychological validation. European Child \& Adolescent Psychiatry, 9(Suppl. 2), 87-101. doi:10.1007/ s007870070012

Hoeft, F., McCandliss, B. D., Black, J. M., Gantman, A., Zakerani, N., Hulme, C., Lyytinen, H., \& Gabrieli, J. D. E. (2011). Neural systems predicting long-term outcome in dyslexia. Proceedings of the National Academy of Sciences, 108, 361-366. doi:10.1073/pnas.1008950108

Iuculano, T., Tang, J., Hall, C. W. B., \& Butterworth, B. (2008). Core information processing deficits in developmental dyscalculia and low numeracy. Developmental Science, 11, 669-680. doi:10.1111/j.1467-7687.2008.00716.x

Karande, S. (2005). Specific learning disability: The invisible handicap. Indian Pediatrics, 42, 315319.

Kosc, L. (1974). Developmental dyscalculia. Journal of Learning Disabilities, 7, 77-164. doi:10.1177/002221947400700309

Koumoula, A., Tsironi, V., Stamouli V., Bardani, I., Siapati, S., Graham-Pavlou, A., Kafantaris, I., ... von Aster, M. (2004). An epidemiological study of number processing and mental calculation in Greek schoolchildren. Journal of Learning Disabilities, 37, 377-388. doi:10.1177/002221940 40370050201

Landerl, K., Bevan, A., \& Butterworth, B. (2004). Developmental dyscalculia and basic numerical capacities: A study of 8-9-year-old students. Cognition, 93, 99-125. doi:10.1016/j.cognition.2003.11.004

Landerl, K., Fussenegger, B., Moll, K., \& Willburger, E. (2009). Dyslexia and dyscalculia: Two learning disorders with different cognitive profiles. Journal of Experimental Child Psychology, 103, 309-324. doi:10.1016/j.jecp.2009.03.006

Landerl, K., \& Kölle, C. (2009). Typical and atypical development of basic numerical skills in elementary school. Journal of Experimental Child Psychology, 130, 546-641. doi:10.1016/j. jecp.2008.12.006 
Machado, A. C., \& Capellini, S. A. (2011). Caracterização do desempenho de crianças com dislexia do desenvolvimento em tarefas de escrita. Revista Brasileira de Crescimento e Desenvolvimento Humano, 21, 132-138.

McCloskey, M. (1992). Cognitive mechanisms in numerical processing: Evidence from acquired dyscalculia. Cognition, 44, 107-157. doi:10.1016/0010-0277(92)90052-J

McCloskey, M., Caramazza, A., \& Basili, A. (1985). Cognitive mechanisms in number processing and calculation: Evidence from dyscalculia. Brain and Cognitive, 4, 171-196. doi:10.1016/02782626(85)90069-7

Mejias, S., Gregoire, J., \& Noel, M. P. (2012). Numerical estimation in adults with and without mathematical learning disabilities. Learning and Individual Differences, 22, 167-170. doi:10.1016/j.lindif.2011.11.012

Moll, K., Göbel, S. M., \& Snowling, M. J. (2014, April 3). Basic number processing in children with specific learning disorders: Comorbidity of reading and mathematics disorders. Child Neuropsychology: A Journal on Normal and Abnormal Development in Childhood and Adolescence. doi:10.1080/09297049.2014.899570

Mousinho, R., Correa, J., \& Mesquita, F. (2010). Perfil da escrita da criança disléxica. In F. C. Capovilla (Ed.), Transtornos de aprendizagem: Progressos em avaliação e intervenção preventiva e remediativa (pp. 190-197). São Paulo, SP: Memnon.

Mundy, E., \& Gilmore, C. K. (2009). Children's mapping between symbolic and nonsymbolic representations of number. Journal of Experimental Child Psychology, 103, 490-502. doi:10.1016/j. jecp.2009.02.003

Oliveira, D. G., Sacchetto, K. K., Ueki, K., Silva, P. B., \& Macedo, E. C. (2011). Análise da produção escrita de crianças com dislexia do desenvolvimento submetidas a intervenção fônica computadorizada. Revista Psicopedagogia, 28, 246-255.

Organização Mundial da Saúde. (1993). Classificação de transtornos mentais e de comportamento da CID-10: Descrições clínicas e diretrizes diagnósticas. Porto Alegre, RS: Artes Médicas.

Price, G. R., Holloway, I., Räsänen, P., Vesterinen, M., \& Ansari, D. (2007). Impaired parietal magnitude processing in developmental dys- calculia. Current Biology, 17, R1042-R1043. doi:10.1016/j.cub.2007.10.013

Rotzer, S., Loenneker, T., Kucian, K., Martin, E., Klaver, P., \& von Aster, M. (2009). Dysfunctional neural network of spatial working memory contributes to developmental dyscalculia. $\mathrm{Neu}$ ropsychologia, 47, 2859-2865. doi:10.1016/j. neuropsychologia.2009.06.009

Rousselle, L., \& Noël, M.-P. (2008). The development of automatic numerosity processing in preschoolers: Evidence for numerosity-perceptual interference. Developmental Psychology, 44, 544-560. doi:10.1037/0012-1649.44.2.544

Rubinsten, O., \& Henik, A. (2006). Double dissociation of functions in developmental dyslexia and dyscalculia. Journal of Educational Psychology, 98(4), 854-867. doi:10.1037/00220663.98.4.854

Salgado, C. A. (2005). Programa de remediaçãofonológica em escolares com dislexia do desenvolvimento (Dissertação de mestrado não-publicada, Faculdade de Ciências Médicas, Universidade Estadual de Campinas, SP, Brasil).

Santos, F. H., Silva, P. A., Ribeiro, F. S., M. C., Dellatolas, G., \& von Aster, M. (2012a). Development of Numerical Representation among Brazilian school-aged children. Jornal Internacional de Estudos em Educação Matemática, 5, 44-64.

Santos, F. H., Silva, P. A., Ribeiro, F. S., Dias, A. L. R. P., Frigério, M. C., Dellatolas, G., \& von Aster, M. (2012b). Number processing and calculation in Brazilian children aged 7-12 years. The Spanish Journal of Psychology, 15, 513525. doi:10.5209/rev_SJOP.2012.v15.n2.38862

Schulte-Körne, G., Warnke, A., \& Remschmidt, H. (2006). Genetics of dyslexia. Zeitschrift fur Kinder- und Jugendpsychiatrie und Psychotherapie, 34(6), 435-444. doi:10.1024/14224917.34.6.435

Shalev, R. S. (2004). Developmental dyscalculia: Review. Journal of Child Neurology, 19, 765-771. doi:10.1016/S0887-8994(00)00258-7

Shalev, R., Manor, O., Amir, N., \& Gross-Tsur, V. (1993). The acquisition of arithmetic in normal children: Assessment by cognitive model of dyscalculia. Developmental Medicine and Child Neurology, 35, 593-601. doi:10.1111/j.1469-8749.1993.tb11696.x 
Silva, P. A., \& Santos, F. H. (2011). Discalculia do Desenvolvimento: Avaliação da representação numérica pela Zareki-R. Psicologia: Teoria e Pesquisa, 27, 169-177. doi:10.1590/S010237722011000200003

Skagerlund, K., \& Träff, U. (2014, March 5). Number processing and heterogeneity of developmental dyscalculia: Subtypes with different cognitive profiles and deficits. Journal of Learning Disabilities.

Snowling, M. J., \& Stackhouse, J. (2004). Dislexia, fala e linguagem: Um manual do profissional. Porto Alegre, RS: Artmed.

StatSoft. (2004). STATISTICA [Data analysis software system], version 7. Retrieved from www. statsoft.com

Steeves, K. J. (1983). Memory as a factor in the computational efficiency of dyslexic children with high abstract reasoning ability. Annals of Dyslexia, 33, 141-152. doi:10.1007/BF02648001

Stein, L. M. (1994). TDE: Teste de Desempenho Escolar: Manual para aplicação e interpretação. São Paulo, SP: Casa do Psicólogo.

Temple, C. M. (1989). Digit dyslexia: A category-specific disorder in developmental dyscalculia. Cognitive Neuropsychology, 6, 93-116. doi:10.1080/02643298908253287

Tressoldi, P. E., Rosatib, M., \& Lucangelic, D. (2007). Patterns of developmental dyscalculia with or without dyslexia. Neurocase: The Neural Basis of Cognition, 13, 217-225. doi:10.1080/13554790701533746

Von Aster, M. G. (2000). Developmental cognitive neuropsychology of number processing and calculation: Varieties of developmental dyscalculia. European Child and Adolescent Psychiatry, 9(Suppl. 2), 41-57. doi:10.1007/s007870070008
Von Aster, M. G., \& Dellatolas, G. (2006). ZAREKI$-R$ : Batterie pour l'évaluation du traitement des nombres et du calcul chez l'enfant. Adaptation francaise [Bateria de Testes Neuropsicológicos para Processamento e Cálculo em Crianças, adaptação francesa]. Paris, France: Les Editions du Centre de Psychologie Appliquée.

Von Aster, M. G., Schweiter, M., \& Weinhold Zulauf, M. (2007). Rechenstörungen bei Kindern. Vorläufer, Prävalenz und psychische Symptome [Discalculia do desenvolvimento: Precursores, prevalência e comorbidade]. Zeitschrift für Entwicklungs Pädagogik, 39, 85-96. doi:10.1026/0049-8637.39.2.85

Von Aster, M. G., \& Shalev, R. S. (2007). Number development and developmental dyscalculia. Developmental Medicine \& Child Neurology, 49, 868-873. doi:10.1111/j.14698749.2007.00868.x

Wechsler, D. (2002). WISC-III: Escala de Inteligência Wechsler para Crianças: Manual (3. ed.). São Paulo, SP: Casa do Psicólogo.

Zuber, J., Pixner, S., Moeller, K., \& Nuerk, H. C. (2009). On the language specificity of basic number processing: Transcoding in a language with inversion and its relation to working memory capacity. Journal of Experimental Child Psychology, 102, 60-77. doi:10.1016/j. jecp.2008.04.003 